\title{
Análisis de las preferencias de los consumidores españoles hacia la miel de producción social y ambientalmente responsable frente a la de Comercio Justo
}

\author{
Sama, C. '; Crespo-Cebada, E. '; Díaz-Caro, C. ${ }^{2}$ y Mesías, F.J. ${ }^{1}$
}

'Departamento de Economía. Universidad de Extremadura. Badajoz. España.

${ }^{2}$ Departamento de Economía Financiera y Contabilidad. Universidad de Extremadura. Cáceres. España.

\section{PALABRAS CLAVE ADICIONALES}

Comercio ético.

Preferencia consumo.

Producción socialmente responsable.

Alimentos.

\section{RESUMEN}

En las últimas décadas se ha producido un notable aumento en la concienciación del consumidor por alimentos de calidad, y producidos de una forma social y ambientalmente responsable. El ámbito agroalimentario presenta uno de los mayores mercados en lo que se refiere a este tipo de producción éticasostenible, como el Comercio Justo, y abre nuevas oportunidades para los productores a través de otros sistemas como la producción social y ambientalmente responsable. El objetivo de este trabajo consiste en determinar los condicionantes en el consumo de alimentos de Comercio Justo, además de la disposición a comprar alimentos social y ambientalmente responsable para la población de Extremadura (España). Para ello se ha aplicado un cuestionario a una muestra representativa $(n=461)$ de la población extremeña en términos de edad y sexo. El análisis empírico consta de un análisis conjunto para determinar las prefe rencias hacia ambos tipos de producto y un modelo probit para determinar qué factores inciden sobre la probabilidad de consumo/disposición a consumir. Los resultados muestran que los consumidores prefieren los dos tipos de producción responsable a la convencional. Asimismo, el perfil de individuo que tiene una mayor probabilidad de consumir alimentos de comercio justo es mujer, con conocimiento previo acerca del Comercio Justo y con sensibilidad hacia los problemas laborales y ambientales. Finalmente, las mujeres, las rentas más altas, y los consumidores sensibilizados y que eran consumidores de Comercio Justo, son las principales características que influyen positivamente sobre la disposición a consumir alimentos social y ambientalmente responsables.

\section{Analysis of Spanish consumers' preferences for socio-environmentally produced honey vs. Fair trade honey}

\section{SUMMARY}

\section{ADDITIONAL KEYWORDS}

Ethical trade.

Consumer's preferences.

Socio-environmentally responsible production. Food.

\section{INFORMATION}

\section{Cronología del artículo.}

Recibido/Received: 06.06.2018

Aceptado/Accepted: 28.11.2019

On-line: 15.10 .2019

Correspondencia a los autores/Contact e-mail:

fimesias@unex.es
In recent decades there has been a notable increase in consumer awareness towards quality foods produced in a socioenvironmentally responsible manner. The agri-food sector presents one of the largest markets in terms of this type of ethical-sustainable production, such as Fair Trade, and this opens up new opportunities for producers through other systems such as socially and environmentally responsible production. The objective of this work is to analyse the determinants of Fair Trade food consumption, as well as the willingness to buy socially and environmentally responsible food for the population of Extremadura (Spain). For this purpose, a questionnaire was applied to a representative sample ( $n=461)$ of the Extremadura population in terms of age and sex. The empirical analysis consisted of a Conioint Analysis in order to determine the preferences for both types of product and a subsequent probit model to determine which factors influence the probability of consumption/willingness to consume. The results show that consumers prefer the two types of responsibly produced food to the conventional one. Likewise, the profile of the individual who has a greater probability of consuming fair trade food is a woman, with prior knowledge about Fair Trade and with sensitivity towards labor and environmental problems. Finally, women, the highest incomes, and consumers who are sensitized and who are consumers of Fair Trade, are the main characteristics that positively influence the willingness to consume socially and environmentally responsible foodstuffs.

\section{INTRODUCCIÓN}

En el contexto actual, con una creciente importancia de la globalización y los movimientos de mercancías a nivel internacional, el grueso de los intercambios se realiza dentro de lo que podríamos denominar "comercio convencional", que está dominado por el libre mercado, y que promueve la competitividad y la obtención del máximo beneficio económico, en muchos 
casos por encima de los derechos de las personas, de las condiciones laborales y de la preservación del medio ambiente. Este sistema genera profundos desequilibrios, no solo en países en desarrollo sino incluso en las economías más potentes donde ciertos colectivos con poco poder negociador pueden ver fuertemente reducidas sus expectativas de generación de ingresos.

Estas desigualdades, junto con la preocupación por la precariedad del bienestar social de los trabajadores y la incidencia negativa en el medio ambiente por parte de la producción, generaron la necesidad de formas alternativas de producción y comercialización, entre las que destaca el Comercio Justo.

Este movimiento surge a mediados del siglo XX con la intención de introducir criterios éticos en el intercambio de mercancías a nivel internacional, especialmente para reducir las desigualdades que se producían en las relaciones comerciales entre los países desarrollados y los países menos desarrollados (Coordinadora Estatal de Comercio Justo 2016). El Comercio Justo crea así unas bases de igualdad y transparencia en las relaciones de trabajo que permiten mejorar las condiciones de vida de los productores, pero también comunicar a los consumidores finales que los productos que adquieren han sido elaborados mediante una producción social y ambientalmente responsable.

El Comercio Justo comenzó vendiendo productos artesanos de decoración, y ya en 1973 empezó a distribuir el primer café producido y comercializado bajo las condiciones de bienestar social y de sostenibilidad, bajo el nombre "Indio Solidarity Coffee", un hito tras el cual las ventas de comercio justo se incrementaron sustancialmente. Posteriormente, en los años 70 y 80 se fueron incorporando gradualmente más alimentos a las tiendas de Comercio Justo como el té, la miel, el cacao, o el azúcar (Coordinadora Estatal de Comercio Justo 2016).

En la actualidad, el Comercio Justo se ha convertido en un movimiento alternativo reconocido mundialmente, que cuenta con más de 2,5 millones de productores en más de 70 países del Sur, superando las 500 organizaciones importadoras y con más de 4.000 tiendas especializadas (Coordinadora Estatal de Comercio Justo 2016).

Considerando el impacto económico, el gasto en productos de Comercio Justo supuso en 2013 un total de 5.500 millones de euros con un incremento del 15\% respecto a 2012 (Fairtrade International 2014) . En España, un mercado menos desarrollado debido a que el Comercio Justo se implantó con posterioridad a otros países europeos, las ventas alcanzaron los 35 millones de euros en 2015, habiéndose duplicado en solo diez años (Coordinadora Estatal de Comercio Justo 2016). Estos ingresos benefician fundamentalmente a los productores y sus familias, que reciben un salario digno y estable, ya que sus ganancias no están tan sujetas a las variaciones de la demanda y la especulación. Pero además el Comercio Justo tiene otros impactos positivos en el medio ambiente (preservación de la tierra, el agua y la biodiversidad, abandono de prácticas perjudiciales, fomento de la agricultura ecológica), la organización política (aplicación de modelos democrá- ticos, participativos y transparentes) y en el entorno, ya que con la prima que reciben las organizaciones productoras éstas desarrollan proyectos educativos, sanitarios, sociales, de infraestructuras, etc. (Stiglitz \& Charlton, 2005).

Desde sus orígenes, este tipo de productos ha estado asociado a los países menos desarrollados en continentes como África, Asia y América del Sur. No obstante, los principios generales del Comercio Justo (salarios equitativos para los trabajadores, distribución justa de beneficios entre productores y distribuidores, buenas prácticas ambientales...), se corresponden con lo que podríamos denominar una "Producción social y ambientalmente responsable" (PSAR), que podría ser desarrollada en determinadas zonas de los países occidentales y a través de las cuales los productores podrían tener acceso a nichos de mercado que garantizaran la rentabilidad de sus actividades y por tanto su persistencia.

Esto se une a recientes movimientos en las cadenas de distribución para reducir la presencia de los alimentos certificados de Comercio Justo utilizando otro tipo de sellos (por ejemplo etiquetados propios del distribuidor) que les permitan ofertar alimentos con un precio más barato pero con el plus de un sistema de producción que aporte al consumidor unos valores de responsabilidad social y ambiental en la producción (Vidal 2017).

En este contexto, este trabajo analiza, por una parte, las preferencias de los consumidores extremeños con respecto a los alimentos de Comercio Justo o de producción social y ambientalmente responsable, y por otra parte, se trata de determinar los factores que influyen en el consumo de alimentos de Comercio Justo y en la disposición a comprar PSAR.

\section{MATERIAL Y MÉTODOS}

Para lograr los objetivos planteados en el trabajo se han aplicado dos metodologías independientes. En primer lugar, la metodología del análisis conjunto que permite determinar las preferencias de los consumidores hacia ambos tipos de producto, así como el potencial de cada uno de ellos. En segundo lugar, se ha utilizado una regresión Probit para determinar el efecto y el signo de las variables socioeconómicas y características de compra sobre la probabilidad de consumo de alimentos de Comercio Justo y la disponibilidad a consumir alimentos de PSAR. Adicionalmente y a partir de la regresión Probit se obtiene la elasticidad de cada variable explicativa con respecto a la probabilidad de consumo de productos de Comercio Justo y de PSAR.

A la hora de desarrollar el estudio se consideró que había que elegir un producto que los entrevistados pudieran identificar sin dificultad con el Comercio Justo, y cuya producción pudieran asociar a aspectos sociales y ambientales. Por ello se eligió la miel como ejemplo de producto a calificar, por ser tradicional, generar grandes beneficios ambientales y sociales en zonas rurales, como sucede en Extremadura y ser un producto habitual en el Comercio Justo. Se definió también como 
forma de presentación el envase de 500 gramos, que es uno de los más habituales en el mercado.

\section{OBTENCIÓN DE DATOS}

Los datos para el estudio han sido obtenidos a partir de un cuestionario realizado a una muestra de 461 consumidores de Extremadura (España), lo que implica un error máximo del $4,6 \%$ con un intervalo de confianza del 95\%. El estudio se desarrolló entre Diciembre de 2016 y Enero de 2017 y los participantes fueron contactados por email, a partir de bases de datos de consumidores creadas en investigaciones anteriores. La Tabla I muestra las características socio-demográficas de la muestra.

Aunque la muestra final está ajustada a la distribución en sexo y edad de la población extremeña (INE 2017), muestra un sesgo hacia personas con estudios universitarios, habitual en estudios online y que puede limitar la interpretación de los resultados.
La elaboración de dicho cuestionario ha sido llevada a cabo a través de Google Forms (www.docs.google. com), siendo este tipo de herramientas online cada vez más utilizada en la investigación por sus ventajas en flexibilidad, rapidez en la recogida de la información y menor coste que con las encuestas tradicionales (Koutsimanis et al. 2012, Kayser et al. 2013), aspectos que compensan sus posibles debilidades como los potenciales sesgos o falta de representatividad de la muestra (Eldesouky et al. 2015)

El cuestionario contenía información relevante acerca del entorno socio-económico de los consumidores -edad (por tramos), sexo, nivel de estudios, nivel de renta del hogar (por tramos)- además de variables relacionadas con el nivel de conocimiento y características del consumo de los alimentos de Comercio Justo, así por ejemplo el lugar y la frecuencia de compra, etc. El cuestionario recogía también cuestiones relacionadas con las preferencias del consumo, lo cual ha permitido llevar a cabo el análisis de los determinantes del con-

Tabla I. Estadísticas descriptivas de la muestra $(n=461)$ (todas las variables son dummy) (Descriptive Statistics of the sample $(n=461)$ (all variables are dummy variables)).

\begin{tabular}{|c|c|c|c|}
\hline Variable & Media & Desviación estándar & Definición de variables \\
\hline Consumo & 0,4381 & 0,4967 & $\begin{array}{l}\text { Toma el valor } 1 \text { si es consumidor tiene una frecuencia media-alta de compra } \\
\text { de alimentos de Comercio Justo, y el valor } 0 \text { en caso que consuma menos de } \\
\text { un producto al año o nunca. }\end{array}$ \\
\hline Disposición a consumir & 0,4685 & 0,4995 & $\begin{array}{l}\text { Toma el valor } 1 \mathrm{si} \text { es consumidor está dispuesto a comprar o a pagar un } \\
\text { sobrecoste por los alimentos de PSAR y toma el valor } 0 \text { en caso contrario. } \\
\text { Construida a partir de la combinación de dos preguntas sobre la disponibilidad } \\
\text { a consumir y a pagar un sobrecoste por este tipo de productos. }\end{array}$ \\
\hline Universitario & 0,7592 & 0,4280 & $\begin{array}{l}\text { Toma el valor } 1 \mathrm{si} \text { el consumidor tiene estudios universitarios y valor } 0 \text { en } \\
\text { caso de estudios inferiores (primarios, bachillerato y formación profesional). }\end{array}$ \\
\hline Renta 1 & 0,2255 & 0,4184 & \multirow{3}{*}{$\begin{array}{l}\text { Definidas de la siguiente forma: renta } 1 \text { para niveles de renta del hogar inferior } \\
\text { a } 1.500 \text { euros al mes; renta } 2 \text { para el tramo de renta } 1.500-2.800 \text { euros y renta } \\
3 \text { para las rentas superiores a } 2.800 \text { euros al mes. }\end{array}$} \\
\hline Renta 2 & 0,4316 & 0,4958 & \\
\hline Renta 3 & 0,3427 & 0,4751 & \\
\hline Conprador habitual & 0,3145 & 0,4648 & $\begin{array}{l}\text { Toma el valor } 1 \mathrm{si} \text { el consumidor es quien realiza la compra en el hogar habi- } \\
\text { tualmente, siendo dicho valor } 0 \text { en caso contrario }\end{array}$ \\
\hline Edad 1 & 0,2212 & 0,4155 & \multirow{3}{*}{$\begin{array}{l}\text { Toman el valor } 1 \text { si la edad del consumidor se encuentra en los intervalos } \\
\text { siguientes: edad 1: entre } 18 \text { y } 30 \text { años; edad } 2 \text { : entre } 31 \text { y } 50 \text { años; edad } 3 \text { : } \\
\text { más de } 50 \text { años. }\end{array}$} \\
\hline Edad 2 & 0,3535 & 0,4785 & \\
\hline Edad 3 & 0,4251 & 0,4949 & \\
\hline Sexo & 0,5509 & 0,4979 & Toma el valor 1 si el consumidor es mujer y 0 si es hombre. \\
\hline Supermercados & 0,2537 & 0,4356 & $\begin{array}{l}\text { Toma el valor } 1 \text { si el consumidor lleva a cabo la compra de productos de } \\
\text { Comercio Justo en supermercados y } 0 \text { en caso contrario. }\end{array}$ \\
\hline Tiendas especializadas & 0,2125 & 0,4095 & $\begin{array}{l}\text { Toma el valor } 1 \text { si el consumidor realiza la compra de productos de Comercio } \\
\text { Justo en tiendas especializadas y } 0 \text { en caso contrario. }\end{array}$ \\
\hline Conocimiento & 0,3383 & 0,4736 & $\begin{array}{l}\text { Toma el valor } 1 \text { si el consumidor manifiesta estar sensibilizado/a con proteger } \\
\text { los derechos de los trabajadores y el medio ambiente y } 0 \text { en caso contrario. } \\
\text { Variable construida a partir de información de dos preguntas del cuestionario, } \\
\text { en las que se preguntaba si conocían los productos de Comercio Justo y los } \\
\text { logos relacionados (Fairtrade, World Fair Trade Organization, Fairtrade lbérica). } \\
\text { A partir de dicha información se construye una variable dummy que toma el } \\
\text { valor } 1 \text { si el individuo manifiesta conocer los productos de Comercio Justo o } \\
\text { los logos relativos a este tipo de mercado y } 0 \text { en caso contrario }\end{array}$ \\
\hline Sensibilización & 0,2673 & 0,4430 & $\begin{array}{l}\text { Toma el valor } 1 \text { si el consumidor manifiesta que no ha consumido productos } \\
\text { de Comercio Justo porque le resulta complicado encontrarlos en el mercado, } \\
0 \text { en caso contrario. }\end{array}$ \\
\hline Dificultad & 0,1561 & 0,3634 & $\begin{array}{l}\text { Toma el valor } 1 \text { si el consumidor manifiesta que no ha consumido productos } \\
\text { de Comercio Justo porque le resulta complicado encontrarlos en el mercado, } \\
0 \text { en caso contrario. }\end{array}$ \\
\hline
\end{tabular}


sumo de productos de origen de Comercio Justo frente a los de PSAR.

Una versión piloto del cuestionario fue enviada a 12 consumidores (no incluidos en la muestra final) para comprobar la relevancia de las preguntas incluidas, así como para detectar posibles errores en la redacción.

Dado que el concepto era novedoso para los consumidores, se incluía una definición de los alimentos de producción social y ambientalmente responsable en el cuestionario antes de las preguntas sobre preferencias del consumidor "alimentos producidos con materias primas de países como España y que hayan sido producidos con criterios social y ambientalmente responsables (salarios dignos a los trabajadores, buenas condiciones laborales, respeto por el medio ambiente, etc.), identificándose con un etiquetado diferenciado".

\section{ANÁlISIS CONJUNTO}

Para el estudio de las preferencias de los consumidores extremeños hacia los alimentos producidos de manera social y ambientalmente responsable se decidió utilizar métodos de preferencias declaradas, ya que se refieren a situaciones donde la elección del encuestado se realiza en contextos de mercados hipotéticos (Jaeger \& Rose 2008).

Dentro de estos, se ha elegido la metodología del análisis conjunto porque permite determinar la importancia relativa de los atributos y niveles que definen un producto, así como el valor económico que el consumidor otorga a la presencia o ausencia de dichos niveles. Como consecuencia de su funcionalidad y practicidad es una técnica ampliamente utilizada en los estudios de preferencias en el sector agroalimentario (Mesias et al. 2013, García-Torres et al. 2016, Donadini et al. 2016, Heide \& Olsen 2017, Endrizzi et al. 2015, Bernabéu \& Díaz 2016).

En el análisis conjunto se describe un producto por medio de los atributos (que presentan además varios niveles) que lo caracterizan y se asume que, a la hora de comprar, el consumidor evalúa la utilidad de la combinación de atributos/niveles, reflejando su elección su priorización subjetiva. Se pide por ello a los entrevistados que, de entre la serie de alternativas que se les presentan (combinaciones de los niveles de los atributos), realicen una clasificación/calificación según sus preferencias hacia cada producto.

Para la selección de atributos se partió de una revisión bibliográfica de distintos trabajos sobre preferencias de consumidores hacia miel (Gyau et al. 2014, Juma et al. 2016, Pocol \& Marghitas 2008, Cosmina et al. 2016, Centre for the Promotion of Imports from Developing Countries (CBI) 2016, Murphy et al. 2000), tras la cual se seleccionaron los atributos y niveles que se muestran en la Tabla II.

La inclusión del atributo "Tipo de Producción" y sus niveles venía dada por los objetivos del estudio. Con respecto al atributo "Origen", se consideró que su inclusión podía tener especial relevancia, dada la relación entre los productos de Comercio Justo y unos orígenes geográficos específicos. En este sentido el nivel "loca/regional" permitía evaluar la aceptación de los alimentos de PSAR por contraposición con los de Comercio Justo, originarios de "países menos desarrollados". La selección del nivel "China, Estados Unidos..." se ha debido a que son los principales países exportadores de miel a España, por lo que son los orígenes de buena parte del producto consumido por los encuestados.

El precio fue incluido en el estudio debido a la amplia variación de precios entre las distintas mieles que se encuentran en el mercado, y que hacía suponer que para ciertos consumidores el precio sería relevante en su elección. Además, este atributo permite realizar una cuantificación en términos monetarios del paso de un nivel de un atributo a otro, es decir, permite representar la utilidad generada por un nivel de un atributo en términos económicos. Los niveles seleccionados para representar el precio, son valores reales que se encuentran en el mercado para tarros de miel de $500 \mathrm{~g}$, variando los niveles desde "3,3€/500g", "5,5€/500g", hasta "7,5€/500g". Finalmente, la producción ecológica se tuvo en cuenta debido a su frecuente asociación con el Comercio Justo.

Las distintas combinaciones de los niveles de la Tabla 2, a través de un diseño ortogonal, dieron lugar a los nueve estímulos finales presentados a los consumidores, que debían puntuarlos en función de su preferencia. La Figura 1 presenta un modelo del producto presentado a los consumidores.

El análisis de los datos se realizó mediante el módulo Conjoint del paquete estadístico SPSS 21, considerando, para los atributos categóricos, que a priori no hay relaciones entre los distintos niveles de los atributos y la utilidad que el consumidor extrae de ellos. Este modelo, denominado part-worth, es según (Green \& Srinivasan 1978) el indicado para variables categóricas. No obstante, se fijó una relación lineal decreciente entre utilidad y precio, modelo que refleja el comportamiento habitual del consumidor.

El modelo se estimó por medio de una regresión por mínimos cuadrados ordinarios, siendo ese el método más utilizado (Wittink \& Cattin 1989). La regla de composición adoptada en este estudio es la aditiva, sin considerar interacción alguna, ya que, según (Hair et al. 1999) este modelo explica hasta un $80-90 \%$ de la variación de la preferencia en casi todos los casos, siendo suficiente para la mayoría de las aplicaciones. Además, muchas veces, la adición de los términos de interacción al modelo reduce el poder predictivo porque la disminución en eficiencia estadística (hay que estimar más part-worths) no se ve compensada por los incrementos en predicción que se obtienen de las interacciones.

Este modelo supone que las preferencias de los entrevistados se obtienen como agregación de las puntuaciones individuales de cada estimulo o atributo (Steenkamp 1987), de tal manera que:

(1)

$$
\text { Valoración }=\alpha_{0}+\sum_{i=1}^{3} \beta_{1 i} D_{1 i}+\sum_{j=1}^{3} \beta_{2 j} D_{2 j}+\sum_{k=1}^{2} \beta_{3 k} D_{3 k}+\sum_{l=1}^{3} \beta_{4 l} D_{4 l}
$$


Donde $\alpha 0$ es el intercepto o término constante, $\beta 1 \mathrm{i}$, $\beta 2 \mathrm{j}, \beta 3 \mathrm{ik}, \beta 4 \mathrm{l}$ son los coeficientes asociados a los niveles $\mathrm{i}(\mathrm{i}=1,2,3) ;(\mathrm{j}=1,2,3) ;(\mathrm{k}=1,2)$ y $(1=1,2,3)$ de los respectivos atributos Tipo de producción (1), Origen (2), Ecológico (3) y Precio (4), siendo las variables D1i, D2j, D3k y D4l las variables dummy de cada uno de los cuatro atributos considerados en este caso.

\section{ANÁLISIS PROBIT}

Posteriormente, en una segunda etapa, se realizaron dos estimaciones mediante un modelo probit para determinar la influencia de las diferentes características socio-económicas y de sensibilización del individuo, así como otros factores asociados al proceso de compra. Se decidió utilizar un modelo probit, ya que la distribución normal del término de error es la más frecuentemente asumida en análisis de consumo. Se realizan, por tanto, dos estimaciones, siendo la variable dependiente en cada una de ellas la probabilidad de consumir alimentos de Comercio Justo y de PSAR, respectivamente. Entre las variables independientes se han considerado la información socio-económica de los consumidores (edad, sexo, nivel de estudios, renta) y algunos condicionantes en el proceso de compra (ser comprador habitual, lugar de compra, facilidad de acceso a dichos productos en el mercado), así como información relativa al conocimiento y la sensibilización del consumidor hacia este tipo de productos. De esta forma es posible considerar que los consumidores pueden responder que sí consumen $(\mathrm{Y}=1)$ o que no consumen $(\mathrm{Y}=0)$, y que la decisión es explicada por un conjunto de factores $X^{\prime}$, tal que:

$$
\operatorname{Pr}\left(Y=1 \mid X_{i}\right)=F\left(X_{i}, \beta_{i}\right)
$$

$$
\operatorname{Pr}\left(Y=0 \mid X_{i}\right)=1-F\left(X_{i}, \beta_{i}\right)
$$

\begin{tabular}{|c|c|}
\hline Atributos & Niveles \\
\hline \multirow{3}{*}{ Tipo de producción } & Comercio Justo \\
\hline & Social y Ambientalmente Responsable \\
\hline & Convencional \\
\hline \multirow{3}{*}{ Origen } & Local/regional \\
\hline & China, Estados Unidos... \\
\hline & Países menos desarrollados \\
\hline \multirow{2}{*}{ Ecológico } & Sí \\
\hline & No \\
\hline \multirow{3}{*}{ Precio } & $3,5 € / 500 \mathrm{gr}$ \\
\hline & $5,5 € / 500 \mathrm{gr}$ \\
\hline & $7,5 € / 500 \mathrm{gr}$ \\
\hline
\end{tabular}

Donde el parámetro $\beta_{i}$ muestra el efecto en el cambio de $X$ sobre la probabilidad de consumir. Si se supone una función de distribución acumulada normal dada por , la expresión del modelo probit toma la siguiente expresión:

(4)

$$
\operatorname{Pr}\left(Y=1 \mid X_{i}\right)=\int_{-\infty}^{X^{\prime} \beta_{i}} \Phi(\mathrm{t}) d t=\Phi\left(\mathrm{x}^{\prime} \beta_{i}\right)
$$

Asimismo, es posible estimar los efectos marginales, de cada variable independiente como muestra la expresión (5):

(5)

$$
\frac{\partial Y}{\partial X}=\Phi\left(\mathrm{x}^{\prime} \beta_{i}\right) \times \beta_{i}
$$

A partir de la expresión (5) se pueden obtener los valores de las elasticidades para cada de las diferentes variables independientes. Los cálculos llevados a cabo para estimar la probabilidad de consumo se han obtenido mediante el programa estadístico Stata v14. Con el comando probit se han estimado las regresiones probabilísticas y el comando mfx se ahn estimado las elasticidades $d_{(\log y) / d\left(\log x_{i}\right)}(c$ comando eyex) en la media de las observaciones.

Los modelos probabilísticos que se propone para la estimación empírica se muestra a continuación:

(6)

$$
\begin{aligned}
& \text { Ocasionales }_{i}=\beta_{0}+\beta_{1} \text { Universitario }_{i}+\beta_{2} \text { Renta }_{i}+\beta_{3} \text { Renta }_{i}+\beta_{4} \text { Comprador }_{i}+ \\
& \beta_{5} \text { Edad }_{i}+\beta_{6} \text { Edad }_{3}+\beta_{7} \text { Sexo }_{i}+\beta_{8} \text { Supermercado }_{i}+\beta_{9} \text { Especializadas }_{i}+ \\
& \beta_{10} \text { Conocimiento }_{i}+\beta_{11} \text { Sensibilización }_{i}+\beta_{12} \text { Dificultad }_{i}+\epsilon_{i}
\end{aligned}
$$

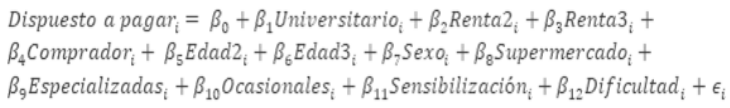

\section{RESULTADOS Y DISCUSIÓN}

A continuación, en la Tabla III, se muestran los resultados del análisis conjunto. Dicha tabla consta de cuatro columnas. La primera y segunda columnas hacen referencia al atributo y niveles del mismo en cada caso: origen del producto (local/regional, procedencia de países en vías de desarrollo o de países desarrollados como China y EE.UU.); si el producto es de producción ecológica o no; el tipo de producción (convencional, comercio justo y PSAR) y finalmente el precio, como referencia del sobre-coste que el consumidor estaría dispuesto a pagar. La tercera y cuarta columnas reflejan la utilidad de cada nivel de atributo y la importancia relativa de cada atributo respectivamente.

En la Tabla III se observa que el origen es el atributo más valorado por los consumidores, con una importancia relativa del 39,17\%, seguido del precio y el tipo de producción, ambos con un peso superior al $20 \%$. 
Tabla III. Estimación Análisis Conjunto (Conjoint analysis estimation)

\begin{tabular}{|c|c|c|c|}
\hline Atributo & Nivel & Utilidad & $\begin{array}{l}\text { Importancia } \\
\text { relativa (\%). }\end{array}$ \\
\hline \multirow{3}{*}{$\begin{array}{l}\text { Tipo de } \\
\text { Producción }\end{array}$} & Convencional & $-0,431$ & \multirow{3}{*}{20,55} \\
\hline & Comercio Justo & 0,197 & \\
\hline & $\begin{array}{c}\text { Social y ambientalmente } \\
\text { responsable }\end{array}$ & 0,235 & \\
\hline \multirow{3}{*}{ Origen } & Regional/local & 0,825 & \multirow{3}{*}{39,17} \\
\hline & $\begin{array}{l}\text { Países menos desarro- } \\
\text { llados }\end{array}$ & 0,138 & \\
\hline & China, Estados Unidos... & $-0,964$ & \\
\hline \multirow{2}{*}{ Ecológico } & Sí & 0,373 & \multirow{2}{*}{16,39} \\
\hline & No & $-0,373$ & \\
\hline \multirow{3}{*}{ Precio } & $3,5 € / 500 \mathrm{~g}$ & $-0,947$ & \multirow{3}{*}{23,87} \\
\hline & $5,5 € / 500 \mathrm{~g}$ & $-1,488$ & \\
\hline & $7,5 € / 500 \mathrm{~g}$ & $-2,030$ & \\
\hline \multicolumn{2}{|c|}{ Pearson's r: 0.998} & \multicolumn{2}{|c|}{ Kendall's tau: 1.000} \\
\hline
\end{tabular}

En relación al tema fundamental de este trabajo se aprecia que los consumidores prefieren los dos tipos de producción responsable (Comercio Justo y PSAR) frente a la convencional, resultado que está en consonancia con otros trabajos como el de (Kimura et al. 2012) en Japón o los de (De Pelsmacker et al. 2005) y (Rousseau 2015) en Bélgica, donde el Comercio Justo también era preferido frente a la producción convencional.

Tabla IV. Modelo Probit para el consumo de alimentos de Comercio Justo (Probit model for the consumption of Fair Trade food).

\begin{tabular}{|c|c|c|}
\hline Variable & $\begin{array}{l}\text { Coeficiente Probit } \\
\text { (Error estándar) }\end{array}$ & $\begin{array}{l}\text { Elasticidades } \\
\text { (Error estándar) }\end{array}$ \\
\hline Universitario & $-0,3284(0,2515)$ & $-0,2559(0,1956)$ \\
\hline Renta 2 & $0,1723(0,2467)$ & $0,074398(0,1092)$ \\
\hline Renta 3 & $0,0625(0,2630)$ & $0,0220(0,9265)$ \\
\hline Comprador habitual & $0,4757^{\star *}(0,2227)$ & $0,3346^{* *}(0,1601)$ \\
\hline Edad 2 & $-0,3178(0,2578)$ & $-0,1153(0,0949)$ \\
\hline Edad 3 & $0,1124(0,2440)$ & $0,04882(0,1057)$ \\
\hline Sexo & $0,4938^{* * *}(0,1894)$ & $0,7323^{* *}(0,2875)$ \\
\hline Supermercados & $1,9130^{\star * *}(0,2253)$ & $0,4983^{* * *}(0,0607)$ \\
\hline $\begin{array}{l}\text { Tiendas } \\
\text { especializadas }\end{array}$ & $1,2071^{* * *}(0,2136)$ & $0,2634^{\star * *}(0,0527)$ \\
\hline Conocimiento & $0,9954^{* * *}(0,2302)$ & $0,6760^{\star * *}(0,1707)$ \\
\hline Sensibilización & $0,8834^{* * *}(0,2023)$ & $0,2419^{* * *}(0,0561)$ \\
\hline Dificultad & $-1,0365^{\star \star \star}(0,3287)$ & $-0,1662^{\star * *}(0,0566)$ \\
\hline Constante & $-2,6374(0,4546)$ & \\
\hline Log Likelihood & $-133,1818$ & \\
\hline Pseudo $\mathrm{R}^{2}$ & 0,5778 & \\
\hline
\end{tabular}

Archivos de zootecnia vol. 68, núm. 264, p. 500.
El hecho de que la producción "social y ambientalmente responsable" presente mayor utilidad que el "comercio justo" indica que, aunque ambos conceptos son atractivos para los consumidores (la utilidad de ambos es positiva, mientras que la utilidad para la producción convencional es negativa) la PSAR es ligeramente preferida, lo que está en consonancia con resultados de otros trabajos, como los de (Engel 2011) o (Aoki et al. 2017), y que indican el menor altruismo de los consumidores de países desarrollados, que buscan los beneficios directos (mejores sistemas de producción, menos contaminación...) sin estar tan interesados en los beneficios a las comunidades en países en desarrollo que genera el Comercio Justo.

Con respecto al origen, en aquellos estudios de preferencias sobre alimentos de Comercio Justo que consideran este atributo, el origen local suele ser preferido a la importación (Aoki et al. 2017, Kimura et al. 2012, Onozaka \& McFadden 2011), aunque estos estudios no diferencian entre países menos desarrollados y otros países. El resultado de nuestro estudio, no obstante, al diferenciar entre dos orígenes no locales claramente diferenciados, permite ahondar en el comentario anterior acerca de la filantropía de los consumidores. No obstante, las preferencias por el origen local podrían estar también ligadas a las preocupaciones y creencias de los consumidores por el impacto ambiental de la producción de alimentos (Tobler et al. 2011, Van Loo et al. 2014).

Otro aspecto destacable es la mayor utilidad media que se otorga a la certificación ecológica frente al tipo de producción de Comercio Justo o de PSAR, lo que está en consonancia con trabajos como los de (Van Loo et al. 2014) y (Aoki et al. 2017) que encontraron que la producción ecológica es preferida al Comercio Justo en USA y Japón, aunque contrasta con los resultados de otros autores (Onozaka \& McFadden 2011, Rousseau 2015, Sepúlveda et al. 2016) también en países desarrollados. Estos resultados divergentes podrían estar relacionados con los distintos productos estudiados, que varían desde café o chocolate, que se producen en países menos desarrollados, a manzanas o miel, que pueden ser originarios de países occidentales.

El interés de los consumidores en los dos conceptos de producción "responsable" justifica el ahondar en el análisis para determinar si las distintas variables socioeconómicas y características de compra inciden en la actual probabilidad de consumo de alimentos de Comercio Justo y en la futura disponibilidad a consumir alimentos de producción social y ambientalmente responsable cuando estos se encuentren en el mercado. Así, la Tabla IV muestra los resultados de la estimación probit, considerando como variable dependiente el consumo de productos de Comercio Justo. La segunda columna de dicha tabla refleja el efecto de cada variable sobre la probabilidad de consumo de alimentos de Comercio Justo y la tercera representa la elasticidad de cada variable en relación al consumo de dichos productos.

La Tabla IV pone de manifiesto que las mujeres presentan una mayor propensión al consumo de Comercio 
Justo que los hombres, siendo la elasticidad de dicha variable de $0,73 \%$.

Asimismo, las variables de sensibilización del consumidor presentan una influencia positiva y significativa sobre la probabilidad de compra de productos de Comercio Justo. Así, la variable "conocimiento" presenta una de las elasticidades más elevadas 0,67\%, indicando que el conocimiento de estos productos o los logos relacionados con el Comercio Justo aumenta la probabilidad de consumo de los mismos, también los consumidores que han manifestado tener cierta sensibilidad hacia las condiciones sociales y medioambientales de los trabajadores y del entorno, que viene recogido por la variable sensibilidad, presentan una elasticidad positiva y significativa del 0,24\%. Además, la dificultad para encontrar dichos productos en el mercado afecta significativamente de forma negativa sobre la probabilidad de consumo de productos de Comercio Justo, con una elasticidad del $-0,17 \%$.

Por otra parte, las variables que reflejan el lugar de compra presentan ambas una influencia positiva y significativa sobre la probabilidad de consumo de productos de Comercio Justo, siendo su elasticidad de $0,50 \%$ para la compra en supermercados y de $0,26 \%$ para las tiendas especializadas, por lo que parece indicar que el establecimiento de compra supone una característica relevante en el proceso de decisión de compra de estos productos. Finalmente, ser comprador habitual en el hogar también supone un efecto positivo

Tabla V. Modelo Probit para la disposición a consumir alimentos de producción social y ambientalmente responsable (Probit model for the willingness to consume socio-environmentally produced food).

\begin{tabular}{lcc}
\hline Variable & $\begin{array}{c}\text { Coeficiente Probit } \\
\text { (Error estándar) }\end{array}$ & $\begin{array}{c}\text { Elasticidades } \\
\text { (Error estándar) }\end{array}$ \\
\hline Consumo & $0,6173^{*}(0,3242)$ & $0,2762^{*}(0,1455)$ \\
Universitario & $0,2254(0,1702)$ & $0,1465(0,1109)$ \\
Renta 2 & $0,1958(0,1669)$ & $0,0720(0,0616)$ \\
Renta 3 & $0,3339^{*}(0,1807)$ & $0,0980(0,0533)$ \\
Comprador habitual & $-0,0597(0,1520)$ & $0,0350(0,0892)$ \\
Edad 2 & $0,1793(0,1853)$ & $0,0542(0,0561)$ \\
Edad 3 & $0,0681(0,1818)$ & $0,0546(0,0558)$ \\
Sexo & $0,4380^{* * *}(0,1297)$ & $0,5911^{* * *}(0,2831)$ \\
Supermercados & $-0,2925(0,2979)$ & $-0,0635(0,0647)$ \\
Tiendas especializadas & $-0,3702(0,3007)$ & $-0,0673(0,0547)$ \\
Conocimiento & $0,2807(0,1719)$ & $0,1595(0,0978)$ \\
Sensibilización & $0,3480^{* *}(0,1691)$ & $0,0795^{\star *}(0,0388)$ \\
Dificultad & $0,2782(0,1906)$ & $0,0372(0,0256)$ \\
Constante & $-1,6944(0,3024)$ & \\
Log Likelihood & $-284,5387$ & \\
Pseudo R ${ }^{2}$ & 0,1052 & \\
\hline
\end{tabular}

Significación: ${ }^{*} p<0,1 ;{ }^{* *} p<0,05 ;{ }^{* * *} p<0.01$. y significativo sobre la probabilidad de consumo de dichos productos, siendo la elasticidad de 0,33\%.

Se observa que la variable sexo tiene una influencia sobre el consumo de Comercio Justo, en concreto las mujeres muestran una mayor probabilidad de consumo de dichos productos, lo cual puede deberse a una mayor sensibilidad y preocupación por el entorno y los derechos laborales de las personas (Aoki et al. 2017, Loureiro \& Lotade 2005).

Otra característica destacable es la condición de comprador habitual en el hogar, que presenta una influencia positiva y significativa sobre el consumo de Comercio Justo, lo cual puede deberse a varios factores: un mayor conocimiento de dichos productos, ya que al ser el comprador habitual tiene acceso a más información sobre los productos, incluso, relacionándolo con el sexo, si el comprador habitual presenta un perfil femenino, pudiendo presentar una mayor sensibilidad hacia estos aspectos.

Asimismo, la variable que recoge la sensibilidad de los encuestados hacia los derechos de los trabajadores y el medio ambiente influye de forma positiva y significativa sobre la probabilidad de consumir productos de Comercio Justo (de Ferran \& Grunert 2007, Weber 2011, Zander et al. 2013), lo cual implica un compromiso ético del consumidor y que tiene un fuerte efecto en la formación del comportamiento del consumidor (Andorfer \& Liebe 2015).

Por otra parte, realizar la compra en supermercados o en tiendas especializadas aumenta la probabilidad de comprar este tipo de productos. Este resultado se encuentra en consonancia con los obtenidos para el caso de compradores de café en Francia (de Ferran \& Grunert 2007) y puede atribuirse a la mayor disponibilidad de estos productos en los supermercados y en las tiendas especializadas, representado ambos establecimientos más del 90\% de la cuota de mercado de estos productos (Sama et al. 2017). Este resultado está en consonancia con el hecho de que los encuestados que manifiestan no consumir productos de Comercio Justo indican que se debe fundamentalmente a la dificultad de encontrarlos en el mercado, teniendo en nuestro estudio una influencia negativa y significativa, lo cual parece indicar que a mayor dificultad a la hora de poder localizar productos de Comercio Justo, menor probabilidad de consumo presentarán los consumidores.

Por otra parte, es destacable la alta significación del conocimiento previo acerca del Comercio Justo, sus características o los logos que representan este tipo de producción, y que suponen una mayor probabilidad en el consumo de los mismos (Rousseau 2015, Van Loo et al. 2015, Zander \& Hamm 2010). Este resultado coincide con otros estudios (Bernabéu \& Díaz 2016, Pedregal \& Ozcaglar-Toulouse 2011), que identifican este hecho con la aceptación de los consumidores (el rechazo en caso de falta de conocimiento) a comprar Comercio Justo.

A continuación, la Tabla $\mathbf{V}$, muestra los resultados de la estimación probit considerando como variable dependiente la disposición de los consumidores extre- 
meños a consumir alimentos social y ambientalmente responsables.

Los resultados de la Tabla $\mathbf{V}$ ponen de manifiesto que los consumidores de productos de Comercio Justo tienen una mayor disponibilidad a comprar alimentos de PSAR, con una elasticidad de $0,28 \%$. Al igual que ocurría con la probabilidad de consumo de Comercio Justo, las mujeres presentan una disposición significativamente mayor a comprar productos social y ambientalmente responsables que los hombres, siendo la elasticidad de dicha variable de 0,59\%. Asimismo, la elasticidad de la variable que recoge la sensibilización del consumidor, $0,08 \%$, es significativa y positiva, por lo que aumenta la disposición de compra de alimentos de PSAR cuanto más sensibilizado esté el consumidor por la protección de los derechos de los trabajadores y del medio ambiente.

El hecho de que los actuales consumidores de alimentos de Comercio Justo estén predispuestos a comprar alimentos de PSAR abre interesantes oportunidades para los productores interesados, ya que estos consumidores suelen mostrar una baja lealtad hacia el Comercio Justo (Cailleba \& Casteran 2010), lo que puede facilitar su transición hacia los PSAR a través de acciones de información basadas en las similitudes entre ambos sistemas de producción.

También es relevante el hecho de que las personas con rentas más altas presenten una mayor disposición hacia la compra de este tipo de alimentos, puesto que el precio es una de las principales barreras a la adquisición de productos de Comercio Justo (Cailleba \& Casteran 2010) y se puede esperar el mismo prejuicio hacia los alimentos PSAR.

Finalmente, la sensibilización sigue jugando un papel significativo en este modelo, con sus claros vínculos con el comportamiento del consumidor ya mencionados. Pero además, otros segmentos de consumidores, como los de productos ecológicos, encuentran entre sus motivaciones de compra aspectos como el apoyo a las estructuras de producción locales o el bienestar de los implicados en la producción de alimentos (Zander et al. 2013), características muy cercanas a la PSAR y que podrían por tanto permitir atraer a estos consumidores.

\section{CONCLUSIÓN}

La situación actual en los mercados agroalimentarios abre oportunidades para productos diferenciados que ofrezcan a los consumidores beneficios adicionales, como pueden ser el cumplimiento de estándares éticos relacionados con los sistemas de producción y las condiciones laborales, en lo que se ha venido a denominar "alimentos de producción social y ambientalmente responsable".

La utilización combinada del análisis conjunto y de las regresiones probabilísticas han permitido el estudio de las preferencias de los consumidores extremeños hacia los alimentos de PSAR, comparándolos con los de Comercio Justo y los de producción convencional y profundizando en los determinantes que inciden en el consumo actual de productos de Comercio Justo y en la probabilidad de adquisición futura de los de PSAR.
Aunque los resultados de este estudio pudieran presentar algunas limitaciones derivadas del método de recogida de datos, se ha observado que los consumidores prefieren los dos tipos de producción responsable (Comercio Justo y PSAR) frente a la convencional, aunque la producción "social y ambientalmente responsable" presenta una mayor utilidad que el "comercio justo". Este hecho, unido a la preferencia por orígenes locales abre interesantes oportunidades para los productores de la región

Se ha encontrado también una significativa relación entre el sexo y el consumo de Comercio Justo, siendo las mujeres las que presentan una mayor predisposición, aspecto que se repite también con los alimentos de PSAR. Asimismo, la sensibilización del consumidor hacia las condiciones sociales y medioambientales de los trabajadores y del entorno presenta también una influencia positiva y significativa sobre la probabilidad de compra ambos tipos de productos.

Los resultados de este estudio pueden tener implicaciones para los productores agroalimentarios, ya que revelan la existencia de nichos de mercado que pueden añadir valor a sistemas de producción tradicionales, lo que puede contribuir a un aumento de las rentas agrarias y complementariamente facilitar el mantenimiento de la actividad agraria en zonas rurales.

\section{BIBLIOGRAFÍA}

Andorfer, VA \& Liebe, U 2015, 'Do information, price, or morals influence ethical consumption? A natural field experiment and customer survey on the purchase of Fair Trade coffee', Social Science Research, vol. 52, pp. 330-350.

Aoki, K, Akai, K, \& Ujiie, K 2017, 'A choice experiment to compare preferences for rice in Thailand and Japan: The impact of origin, sustainability, and taste', Food Quality and Preference, vol. 56, pp. 274-284.

Bernabéu, R \& Díaz, M 2016, 'Preference for olive oil consumption in the Spanish local market', Spanish Journal of Agricultural Research, vol. 14, no. 4.

Cailleba, P \& Casteran, H 2010, 'Do Ethical Values Work? A Quantitative Study of the Impact of Fair Trade Coffee on Consumer Behavior', Journal of Business Ethics, vol. 97, no. 4, pp. 613-624.

Centre for the Promotion of Imports from Developing Countries (CBI) 2016, Which trends offer opportunities on the European honey market? Coordinadora Estatal de Comercio Justo 2016, 'El Comercio Justo en España 2015', El comercio justo en España 2015, p. 70.

Cosmina, M, Gallenti, G, Marangon, F, \& Troiano, S 2016, 'Attitudes towards honey among Italian consumers: A choice experiment approach', Appetite, vol. 99, pp. 52-58.

Donadini, G, Fumi, MD, Kordialik-Bogacka, E, Maggi, L, Lambri, M, \& Sckokai, P 2016, 'Consumer interest in specialty beers in three European markets', Food Research International, vol. 85, pp. 301-314.

Eldesouky, A, Pulido, AF, \& Mesias, FJ 2015, 'The Role of Packaging and Presentation Format in Consumers' Preferences for Food: An Application of Projective Techniques', Journal of Sensory Studies, vol. 30, no. 5, pp. 360-369.

Endrizzi, I, Torri, L, Corollaro, ML, Demattè, ML, Aprea, E, Charles, M, Biasioli, F, \& Gasperi, F 2015, 'A conjoint study on apple acceptability: Sensory characteristics and nutritional information', Food Quality and Preference, vol. 40, no. PA, pp. 39-48.

Engel, C 2011 , 'Dictator games: A meta study', Experimental Economics, vol. 14, no. 4, pp. 583-610.

Fairtrade International 2014, 'Annual Report 2013-2014', Fairtrade International | Annual Report. 
de Ferran, F \& Grunert, KG 2007, 'French fair trade coffee buyers' purchasing motives: An exploratory study using means-end chains analysis', Food Quality and Preference, vol. 18, no. 2, pp. 218-229.

García-Torres, S, López-Gajardo, A, \& Mesías, FJ 2016, 'Intensive vs. free-range organic beef. A preference study through consumer liking and conjoint analysis', Meat Science, vol. 114, pp. 114-120.

Green, PE \& Srinivasan, V 1978, 'Conjoint Analysis in Consumer Research: Issues and Outlook', Journal of Consumer Research, vol. 5, pp. 103-123.

Gyau, A, Akalakou, C, Degrande, A, \& Biloso, A 2014, 'Determinants of Consumer Preferences for Honey in the Democratic Republic of Congo', Journal of Food Products Marketing, vol. 20, no. 5, pp. 476-490.

Hair, JF, Anderson, RE, Tatham, RL, \& Black, WC 1999, Análisis multivariante, Prentice Hall, Madrid.

Heide, M \& Olsen, SO 2017, 'Influence of packaging attributes on consumer evaluation of fresh cod', Food Quality and Preference, vol. 60, pp. 9-18.

INE 2017, Censos de población y vivienda, Instituto Nacional de Estadística. INEbase 2011.

Jaeger, S \& Rose, J 2008, 'Stated Choice experimentation, contextual influences and food Choice: A case study', Food Quality and Preference, vol. 19, pp. 539-564.

Juma, CN, Otieno, DJ, Oluoch-Kosura, W, Gyau, A, \& Oduol, JA 2016,'A survey of consumer perceptions and preferences for geographical indication and quality attributes of honey in Kenya', in, 5th International Conference of the African Association of Agricultural Economists, Addis Ababa, Ethiopia

Kayser, M, Nitzko, S, \& Spiller, A 2013, 'Analysis of Differences in Meat Consumption Patterns', International Food and Agribusiness Management Review, vol. 16, no. 2, pp. 43-56.

Kimura, A, Mukawa, N, Yamamoto, M, Masuda, T, Yuasa, M, Goto, S, Oka, T, \& Wada, Y 2012, 'The influence of reputational concerns on purchase intention of fair-trade foods among young Japanese adults', Food Quality and Preference, vol. 26, no. 2, pp. 204-210.

Koutsimanis, G, Getter, K, Behe, B, Harte, J, \& Almenar, E 2012, 'Influences of packaging attributes on consumer purchase decisions for fresh produce', Appetite, vol. 59, no. 2, pp. 270-280.

Van Loo, EJ, Caputo, V, Nayga, RM, Seo, HS, Zhang, B, \& Verbeke, W 2015, 'Sustainability labels on coffee: Consumer preferences, willingness-to-pay and visual attention to attributes', Ecological Economics, vol. 118, pp. 215-225.

Van Loo, EJ, Caputo, V, Nayga, RM, \& Verbeke, W 2014, 'Consumers' valuation of sustainability labels on meat', Food Policy, vol. 49, no. P1, pp. 137-150.

Loureiro, ML \& Lotade, J 2005, 'Do fair trade and eco-labels in coffee wake up the consumer conscience?', Ecological Economics, vol. 53, no. 1, pp. 129-138.

Mesias, FJ, Pulido, F, Escribano, M, Gaspar, P, Pulido, AF, Escribano, A, \& Rodriguez-Ledesma, A 2013, 'Evaluation of new packaging formats for dry-cured meat products using conjoint analysis: An application to dry-cured iberian ham', Journal of Sensory Studies, vol. 28, no. 3, pp. 238-247.
Murphy, M, Cowan, C, O'Reilly, S, \& Henchion, H 2000, 'Consumer Preference for Honey: A Conjoint Approach.', British Food Journal, vol. 102, no. 8, pp. 585-597.

Onozaka, Y \& McFadden, DT 2011, 'Does local labeling complement or compete with other sustainable labels? A conjoint analysis of direct and joint values for fresh produce claim', American Journal of Agricultural Economics, vol. 93, no. 3, pp. 689-702.

Pedregal, VD \& Ozcaglar-Toulouse, N 2011, 'Why does not everybody purchase fair trade products? The question of the fairness of fair trade products' consumption for consumers', International Journal of Consumer Studies, vol. 35, no. 6, pp. 655-660.

De Pelsmacker, P, Driesen, L, \& Rayp, G 2005, 'Do consumers care about ethics? Willingness to pay for fair-trader coffee', The Journal of Consumers Affairs, vol. 13, no. 2, pp. 363-386.

Pocol, CB \& Marghitas, LA 2008, 'A comparison between the segmentation of honey market in Romania and Ireland', Bulletin of University of Agricultural Sciences and Veterinary Medicine Cluj-Napoca. Horticulture, vol. 65, no. 2, pp. 279-282.

Rousseau, S 2015, 'The role of organic and fair trade labels when choosing chocolate', Food Quality and Preference, vol. 44, pp. 92-100.

Sama, C., Crespo-Cebada, E., Díaz-Caro, C., Eldesouky, A., Elghannam, A., \& Mesias, FJ 2017,'Los alimentos de producción social y ambientalmente responsable y su aceptación por los consumidores extremeños', in AE de E Agraria. (ed.), Actas del XI Congreso de la Asociación Española de Economía Agraria., Orihuela- Elche (España).

Sepúlveda, WS, Chekmam, L, Maza, MT, \& Mancilla, NO 2016, 'Consumers' preference for the origin and quality attributes associated with production of specialty coffees: Results from a cross-cultural study', Food Research International, vol. 89, pp. 997-1003.Steenkamp, J-BEM 1987, 'Conjoint measurement in ham quality evaluation', Journal of Agricultural Economics, vol. 38, no. 3, pp. 473-480.

Stiglitz, J.E., and Charlton, A (2005). Fair Trade for all: how trade can promote development. Oxford University Press on Demand.

Tobler, C, Visschers, VHM, \& Siegrist, M 2011 , 'Eating green. Consumers' willingness to adopt ecological food consumption behaviors', Appetite, vol. 57, no. 3, pp. 674-682.

Vidal, J 2017, 'Move by UK supermarkets threatens to bring Fairtrade crashing down', The Guardian.

Weber, JG 2011, 'How much more do growers receive for Fair Tradeorganic coffee?', Food Policy, vol. 36, no. 5, pp. 677-684.

Wittink, DR \& Cattin, P 1989, 'Commercial use of Conjoint Analysis: an update', Journal of Marketing, vol. 53, pp. 91-96.

Zander, K \& Hamm, U 2010, 'Consumer preferences for additional ethical attributes of organic food', Food Quality and Preference, vol. 21, no. 5, pp. 495-503.

Zander, K, Stolz, H, \& Hamm, U 2013, 'Promising ethical arguments for product differentiation in the organic food sector. A mixed methods research approach', Appetite, vol. 62, pp. 133-142. 